in a field which is trying to be patient centred.

\title{
Flexible rather than standardised approaches to communicating risks in health care
}

\section{A Edwards}

\section{A sufficiently flexible approach to risk communication is needed to accommodate a wide range of patient interpretation and preferences for information}

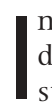
nformed medical decision making depends at least partly on understanding the benefits and harms of different treatment options. This requires clear and relevant risk communication. In practice this means that information should include both harms and benefits where relevant, and be presented honestly-not hiding information thought to be less desirable. Attention should be paid to the potential pitfalls of "framing" — how different formats such as relative and absolute risk can manipulate decisions made. ${ }^{1}$ Certain types of risk information such as "natural frequencies" (for example, 1 in 10) are generally more consistently and accurately interpreted than percentages (for example, 10\%). In percentages, the reference class (population or group to which the figure applies) is often not clearly specified. ${ }^{2}$ For any data, though, there are uncertainties and these should be shared where evident. ${ }^{3}$ There is support for having a range of information formats available (a "toolbox") so that professionals can use the most appropriate one to aid discussions with individual patients. ${ }^{4}$ Such formats may be descriptive, numerical, or graphical. They may include patient narratives of their experiences to convey the pros and cons of decisions made in certain situations. Patient preferences for different formats and levels of detail will vary, and the materials should be there to accommodate this range.

However, the appropriate language for effective risk communication with patients is less clear. Both patients and professionals have difficulty in interpreting, incorporating, and remembering risk information, especially the statistical aspects. ${ }^{5}$ There are wide variations in the interpretation of probabilistic terms with regard to the frequencies they convey. ${ }^{6}$ Few studies of preferences for or understanding of risk information in practice have been done with real patients. Most current literature describes conceptual development or hypothetical scenarios, but people interpret information differently in hypothetical and real settings. ${ }^{1}$ There is a clear need for more research to identify the effects of different formats of risk information and effective ways of communicating such information to patients having to make a relevant health care choice.

Pending the availability of such findings, some authors have proposed the use of standardised "languages" for risk communication. ${ }^{78}$ These aim to encourage professionals to use agreed terms such as "common", "sometimes", or "rare" for risks of certain frequencies. The hope is that, through continued usage, the terms would become increasingly accepted and consistently interpreted. However, there are several potential pitfalls to this approach. People vary in their preferred style of risk information-verbal or descriptive versus more numerical approaches. Standardised terms may also not be sufficiently flexible for the range and complexity of risks encountered in clinical practice. A range of primary care professionals indicated that standardised approaches would be unlikely to help discussions with patients, although there was potential to improve communication between professionals. ${ }^{9}$ The data in the paper by Knapp et $a l^{10}$ published in this issue of QSHC indicate a further difficulty. These authors showed that the severity of the condition adds a further dimension to the variation in interpretation of probabilistic information. The frequency attributed to a "rare" serious side effect was different from a "rare" less serious side effect. Language is also dynamic, an ever-changing phenomenon, and thus perhaps inherently resistant to control. ${ }^{11}$ All these difficulties undermine efforts to standardise the language of risk, a strategy which could be viewed as inherently "top down" or paternalistic
Given this variability of interpretation, changing characteristics of language, and flexibility required for risk communication approaches with individuals, we need broader strategies for enhancing risk communication, whether for individuals or large groups of people. Some lessons can be illustrated by the example of the scare over third generation oral contraceptives in 1995. Poorly communicated risk information led to patient anxiety, loss of confidence in the treatments and the authorities providing the information and, crucially, the discontinuation of medication and unplanned pregnancies. This example informs us about the need for effective, balanced communication of risk information. This would include absolute and possibly relative risk formats, but not relative risk in isolation, and attention to the wider potential pitfalls of framing manipulations such as positive or negative framing (chances of survival or death). ${ }^{1}$ All probabilistic information requires adequate explanation appropriate to the individual. For information that must be disseminated in a uniform way, such as in patient package inserts, this requires a sufficient range of formats-descriptive, numerical, visual-to be likely to meet the preferences of a large proportion of potential readers. ${ }^{12}$ This is challenging, but the contraceptive scare story illustrates the huge price to pay when getting it wrong. When discussing information with individuals, professionals also need to explore the meaning and significance of the risk, and the chances to the individual of beneficial or adverse outcomes from different treatment options. They need to explore people's understanding and reactions to the information, and how it integrates with other influences on health, health care, and decision making such as past experiences, family pressures, and so on.

Risk information is just one of the contributors towards decision making. Efforts to improve it should not bypass or undermine other major contributors such as support from others (family friends, professionals) and the trust that is placed in these people. ${ }^{3}$ Good risk communication is, however, important if people are to be adequately informed and enabled to make decisions about their treatment or care. For this they need sufficient information, tailored and clearly presented in a format or style and level of detail with which they are comfortable to aid their decision making. It should be relevant to their situation, contextualised for prior experiences, and seek to achieve sufficient 
understanding of the condition(s) and risks concerned. There are a number of issues that cause deep seated difficulties for attempts to standardise the language of risk communication. Our approaches to risk communication need to be sufficiently flexible to accommodate the range of patient interpretation and preferences for information.

Qual Saf Health Care 2004;13:169-170. doi: 10.1136/qshc.2004.010850

Correspondence to: Dr A Edwards, Department of Primary Care, Swansea Clinical School, University of Wales Swansea, Singleton Park, Swansea SA2 8PP, UK; a.g.k.edwards@swan. ac.uk

\section{REFERENCES}

1 Edwards A, Elwyn G, Covey J, et al. Presenting risk information: a review of the effects of 'framing' and other manipulations on patien outcomes. J Health Commun 2001;6:61-82.

2 Gigerenzer G, Edwards A. Simple tools for understanding risks: from innumeracy to insight BMJ 2003;327:741-4.

3 Edwards A. Communicating risks: means that patients too have to learn to live with uncertainty. BMJ 2003:327:691-2.

4 Paling J. Strategies to help patients understand risks. BMJ 2003;327:745-8.

5 Lloyd A. The extent of patients' understanding of the risk of treatments. Qual Health Care 2001;10(Suppl I):i14-i18.

6 Cohn LD, Schydlower M, Foley J, et al. Adolescents' misinterpretation of health risk probability expressions. Pediatrics 1995;95:713-6.
7 Calman K, Royston G. Risk language and dialects. BMJ 1997;315:939-42.

8 Paling J. Up to your armpits in alligators: how to sort out what risks are worth worrying about, 1 st ed. Gainesville, FL: Risk Communication and Environmental Institute, 1997.

9 Edwards A, Matthews E, Pill RM, et al Communication about risk: the responses of primary care professionals to standardising the language of risk and communication tools. Fam Pract 1998;15:301-7.

10 Knapp P, Raynor DK, Berry DC. Comparison of two methods of presenting risk information to patients about the side effects of medicines. Qual Saf Health Care 2004; 13:176-80.

11 Edwards A, Pill RM, Stott NCH. Communicating risk: use of standard terms is unlikely to result in standard communication. BMJ 1996:313:1483.

12 Woloshin S, Schwartz L. How can we help people make sense of medical data? Effect Clin Pract 1999:2:176-83.

\section{Safety from numbers: identifying drug related morbidity using electronic records in primary care}

\section{G Elwyn}

\section{The use of electronic clinical data to identify temporal associations between drug prescribing and patient morbidity}

$\mathrm{T}$ he benefits of creating a searchable patient record are slowly emerging, although it is arguable that progress has been significantly delayed by system designs that failed to focus on overall aims. It is well recognised that it is easier to enter data into clinical systems than to analyse them in order to answer questions about quality, care patterns, longitudinal trends, drug interactions, and patient safety. Although clinical information systems have slowly evolved to provide more user friendly interfaces, they still struggle with two important areas: data coding and pattern analysis. The next logical step-to mine datasets and present meaningful data patterns using visualisation techniqueshas hardly been tackled. Nevertheless, researchers are slowly negotiating the rocky path from clinical data to information to knowledge.

An important inherent ability of clinical information systems is to signal possible linkages between events: to alert health professionals to be vigilant or to avoid risk. It has been postulated that it might be possible to analyse the harm that arises as a result of prescribing drugs. If patterns could be recognised, then it might be possible either to avoid the possibility or to build in safety nets to reduce potential problems. Patient datasets could be searched for possible risky combinations (co-morbidities and drug combinations) and actions taken to reduce the chance of harmful outcomes. Another realm of anticipatory care becomes available. The first step is to predict some possible "indicator" associations between prescribing and "harmful events".

In this issue of QSHC Morris and colleagues have adapted work from the US by MacKinnon and Hepler ${ }^{1}$ who found that it was feasible to examine associations between patient morbidity and prescribed drugs by examining electronic clinical records. ${ }^{2}$ The work in North America was done on a hospital database in a managed care organisation, so it is likely that this UK article which used primary care records has a better dataset. It is also likely that the implementation of the quality indicator based contract in general practice ${ }^{3}$ will enhance the accuracy of coding in consultations over the next few years, increasing the chances that this type of work will be easier to conduct.

Three key areas of risk were foundnamely, patients with heart failure or hypertension who use non-steroidal anti-inflammatory drugs, patients who are not monitored when using angiotensin converting enzyme inhibitors, and patients who use regular hypnotic-anxiolytic drugs. The authors avoid claiming that the patterns are evidence of causality-that, for instance, the use of hypnotic-anxiolytic drugs leads directly to hip fractures. Nevertheless, the development of possible temporal indicators and emergent patterns are important findings. Problems identified in the US are found in the UK and a "pareto" principle is demonstrated-a small number of possible interactions are associated with a large percentage $(60 \%)$ of potentially preventable morbidity.

Drug related morbidity attributable to predicted associations was found in $1 \%$ of adult patients in the time frame studied (27 months). It is probable that under-recording in electronic records means that this is an underestimate of incident drug related morbidity. As "during consultation" coding improves, the epidemiological drug morbidity profiles achieved at practice level may well improve and potential benefits be more easily identified. Notice, for instance, the laborious data extraction, data cleaning, and repeated analysis that had to be undertaken to arrive at the results presented by Morris et al.

If this work is replicated, even if only the most common associations are correct, then knowing that these three patient groups are at significantly increased risk should lead to preventative strategies. It is therefore likely that the avoidance of drug related harm by planned review will become a future quality indicator. The authors want to use the results to generate discussion about these possible developments. They do not think that existing databases are accurate enough for comparisons between practices and are wary of such 
potential developments. It is possible, however, to speculate that this type of analysis might occur as the patient record becomes electronic and shared more widely. Could a detailed interrogation of associations between events and antecedent prescribing have medicolegal implications? The validity of the "association indicators" would then become essential.

Extracting and analysing electronic clinical data in order to identify temporal associations between drug prescribing and patient morbidity is an exciting step forward. At the moment it requires database analysts to sift through the codes. But it will get easier. Used in a preventative strategic way to improve patient care, the technique could be a valuable intervention for quality improvement. If, on the other hand, a more inquisitorial use comes about, it might be more difficult to harness the benefits for patients in the long term.

Qual Saf Health Care 2004;13:170-171. doi: 10.1136/qshc.2003.009712
Correspondence to: Professor G Elwyn, Primary Care Research Group, University of Wales Swansea Clinical School, Swansea SA2 8PP, UK; g.elwyn@swansea.ac.uk

\section{REFERENCES}

1 MacKinnon NJ, Hepler CD. Preventable drugrelated morbidity in older adults. 1. Indicator development. J Man Care Pharm 2002;8:365-71.

2 Morris CJ, Rodgers S, Hammersley VS, et al. Indicators for preventable drug related morbidity: application in primary care. Qual Saf Health Care 2004;13:181-5.

3 Shekelle P. New contract for general practitioners. BMJ 2003;326:457-8.

\section{$\mathrm{ECHO}$}

\section{Surgical quality: review of Californian measures}

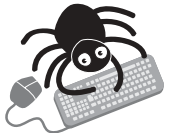

Please visit the Quality and Safety in Health Care website [www. qshc.com] for a link to the full text of this article.

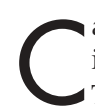

alifornians wanting to select a hospital or surgeon on the basis of publicly available information on quality will find serious shortcomings in the available data.

was the finding from a review of 18 organisations in California, USA reporting 333 measures of healthcare quality. Shortcomings were at several levels, although foremost was that all the organisations examined quality at the hospital level-none at the level of the individual surgeon, group of surgeons or health plan.

For 21 procedures, accounting for $21 \%$ of the surgical procedures, structure, process and outcome measures were looked for by the researchers. Organisations reported structure measures (such as annual hospital volume) for 12 procedures. None reported any process measures. Outcome measures were reported for 19 procedures, the most commonly reported being death in hospital and the major complication rate.

Six of the 10 most common non-obstetric procedures, including hysterectomy and cholecystectomy, had no reported quality measures at all. Furthermore because of the time lag between collecting and reporting data, most current measures reflected care that had been delivered between two and five years previously.

The situation is set to improve by 2005 when three new publicly available quality measures are expected relating to hip fracture, carotid endarterectomy and coronary artery bypass grafting. For the last of these the quality measure will include risk adjusted deaths specific to individual surgeons.

An accompanying commentary commented that there are essentially three problems to the task of improving health care by publishing outcomes from healthcare providers. The first is to find outcomes that provide good comparable information, allowing for differences in case mix and with sufficient power that differences between providers do not arise by chance.

The second is to make sure that this information is used to genuinely improve the quality of care provided by those underperforming - and not just a change in their reporting. Finally, as illustrated by the review from California, the third problem is to find measures that comprehensively capture information about health care which is meaningful to the individual and, importantly, provide this information in a timely way.

\ BMJ 2004;328:152-153 